\title{
Influence of Human Papillomavirus on the Clinical Presentation of Oropharyngeal \\ Carcinoma in the United States
}

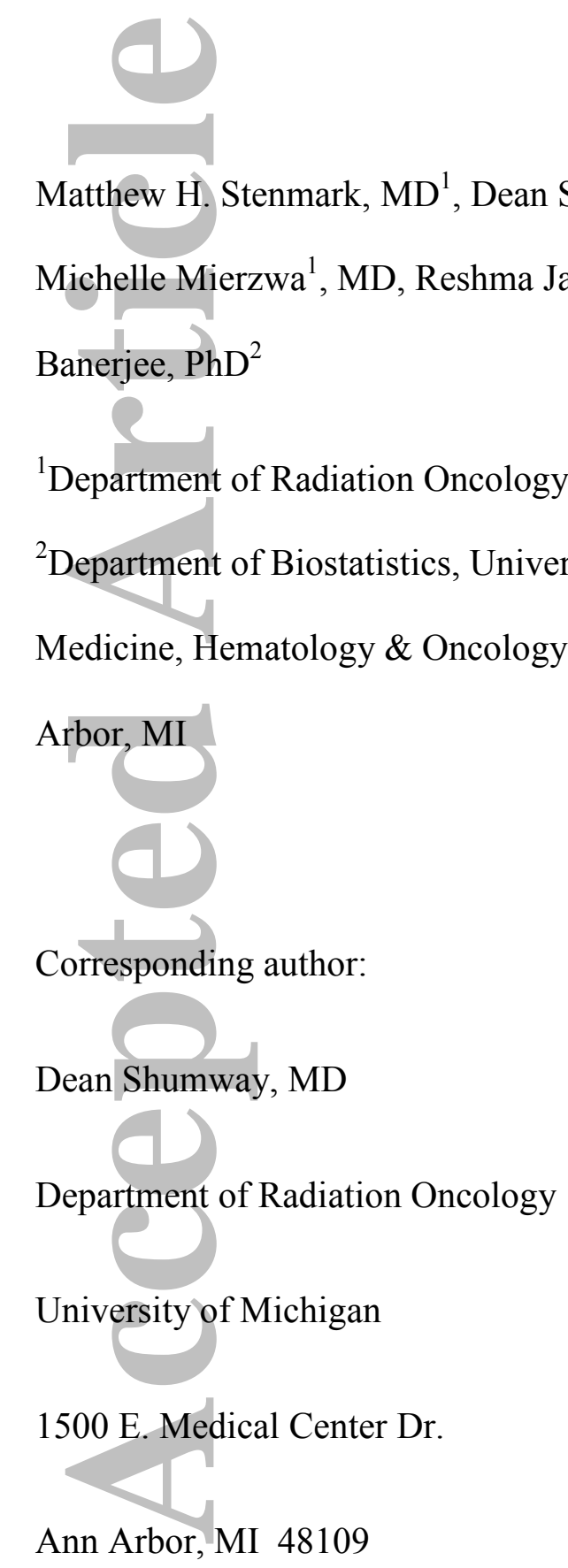

Phone: 734-936-4300

Email: dshumway@umich.edu

This is the author manuscript accepted for publication and has undergone full peer review but has not been through the copyediting, typesetting, pagination and proofreading process, which may lead to differences between this version and the Version record. Please cite this article as doi:10.1002/ lary.26566. 
Funding Sources: Dr. Banerjee's research was partially funded by grant CA 046592 from the National Cancer Institute

Running title: HPV-related oropharynx cancer in the US

Text pages: 18

Tables: 2

Figures: 2

Conflicts of Interest: none

Financial disclosures: none

Keywords: oropharyngeal squamous cell carcinoma, human papillomavirus, HPV, clinical presentation, National Cancer Data Base

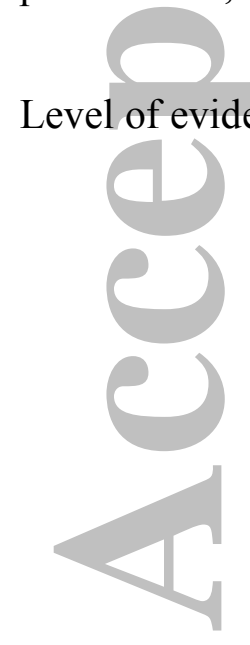

John Wiley \& Sons

This article is protected by copyright. All rights reserved. 


\section{ABSTRACT}

\section{Objective}

Much of what is known about the significance of human papillomavirus (HPV) in oropharyngeal cancer is derived from single-institution retrospective studies, post-hoc analyses of tissue specimens from clinical trials, and tissue bank studies with a small sample size. The objective of this study is to investigate the impact of HPV on the frequency and clinical presentation of oropharyngeal carcinoma in a large, national sample with information from patients who underwent HPV testing.

\section{Study Design}

Retrospective, cross-sectional study.

\section{Methods}

We identified a comprehensive national sample of 8,359 patients with oropharyngeal carcinoma and known HPV status diagnosed between 2010 and 2011 within the National Cancer Data Base. Multivariable logistic regression was used to assess correlates of patient and tumor characteristics on HPV status.

\section{Results}

Among patients with oropharyngeal carcinoma, the frequency of HPV-related squamous cell carcinoma in the United States was $65.4 \%$. HPV-related oropharyngeal carcinoma was associated with younger age, male sex, and white race $(P<0.001)$. Advanced primary tumor stage was associated with HPV-negative disease $(P<0.001)$, while increasing nodal burden was 
associated with HPV-positive disease $(P<0.001)$. Despite less advanced nodal disease, HPVnegative tumors were associated with a higher likelihood of metastasis at presentation $(P<0.001)$.

\section{Conclusion}

HPV now accounts for the majority of newly diagnosed oropharyngeal carcinoma in the United States and is associated with a distinct clinical profile, supporting efforts to re-evaluate the staging and treatment paradigm for HPV-associated oropharyngeal cancer.
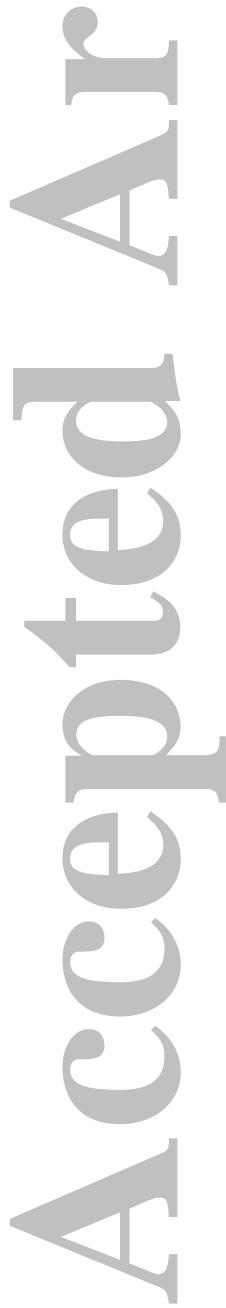


\section{Introduction}

Over the past three decades, the incidence of oral cavity, laryngeal, and hypopharyngeal cancers has been declining in the United States, ${ }^{1}$ concurrent with a decrease in tobacco use over this time frame. In contrast, the incidence of oropharyngeal squamous cell carcinoma (OPC) has been on the rise, predominately among white men less than 60 years of age, both in the United States ${ }^{1}$ and in other economically developed countries in North America ${ }^{2,3}$ and Western Europe., 4-6 Epidemiologic and molecular studies have shown this site-specific increase in oropharyngeal cancer incidence to be attributable to infection with high-risk human papillomavirus (HPV), particularly oncogenic HPV subtype 16, resulting in a distinct neoplastic entity. ${ }^{1,7}$ A recent systematic review comprised predominately of studies from North America and Europe, demonstrated a significant increase in the percentage OPCs attributable to HPV over time, from $40.5 \%$ before 2000 , to $64.3 \%$ between 2000 and 2004 , to $72.2 \%$ between 2005 and $2009 .{ }^{8}$ In addition, retrospective analyses of clinical trial populations from North America, Europe, Australia and New Zealand from 2002 to 2005 report HPV incidence rates of $57.3 \%$ and $63.8 \%$ among 185 and 323 patients with stage III to IV OPC, respectively., ${ }^{9,}$

These same studies show that the presence of HPV is a favorable independent prognostic factor for overall survival. ${ }^{9-12}$ Case studies have also shown that individuals with HPV-associated OPC demonstrate unique clinical and behavioral characteristics, including younger age at presentation, white male predominance, and a greater number of oral sex partners. ${ }^{12-15}$ In contrast, HPV- 
negative OPC is associated with tobacco and alcohol exposure with the strength of the association increasing with the intensity and duration of use. ${ }^{13}$ A difference in the distribution of primary tumor and nodal classification between HPV-positive and HPV-negative carcinomas has also been described.

However, much of what is known about the significance of HPV in oropharyngeal cancer is derived from single-institution retrospective studies, ${ }^{7,13}$ post-hoc analyses of tissue specimens from elinical trials, ${ }^{9-11,16}$ and one study with prospective evaluation of HPV in a subset of 96 patients enrolled on a clinical trial. ${ }^{12}$ These studies are limited by small sample size, restrictive eligibility criteria of prospective trials, and heterogeneity of the study population due to inclusion of multiple primary cancer sites. For example, an analysis of tissue specimens from 271 patients from the Iowa, Hawaii, and Los Angeles SEER regions demonstrated prevalence of HPV-related disease of $72 \%$ during 2000-2004. ${ }^{1}$ Very similar results were observed in an analysis of 203 tonsillar cancer specimens from Sweden. ${ }^{4}$ The generalizability of these studies is limited by their small sample size and nonrepresentativeness of tested patients. Other epidemiologic studies defined "potentially HPV-associated cancers" based on the anatomical subsite of disease, 2, 17, 18 but did not have information on the HPV status of tumor tissues.

While the changing epidemiology of OPC has been well documented, population level data on the frequency and clinical features of HPV-related OPC are lacking. There has not yet been an analysis of HPV-associated OPC in a large, national sample with information from patients who underwent HPV testing. This study seeks to describe the influence of HPV status on clinical presentation on a national level. 


\section{Methods}

\section{Data Source and Study Population}

The National Cancer Data Base (NCDB) is a nationwide, facility-based, oncology database of the American College of Surgeons Commission on Cancer $(\mathrm{CoC})$ that currently captures $70 \%$ of all new cancer diagnoses in the United States annually from over 1,400 Commission-accredited cancer programs across a diverse range of geographic regions. Data are abstracted by trained tumor registrars and reported using national established protocols coordinated though the North American Association of Central Cancer Registries. Ongoing data quality-assurance assessments are performed using both automated electronic checks and external site surveyors. ${ }^{19}$ As no patient, physician, or hospital identifiers were examined in this study, exemption was granted by the University of Michigan Institutional Review Board.

The International Classification of Disease for Oncology, third edition (ICD-0-3), was used to identify 22,494 patients within the NCDB who were diagnosed between January 1, 2010 and December 31, 2011 with primary carcinoma of the oropharynx. The data query was restricted to 2010-2011 to coincide with availability of HPV data from the NCDB. Cases were identified using ICD-0-3 topography codes C01.9, C02.4 (base of tongue), C09.0-09.1, C09.8-09.9 (tonsil), C05.1-05.2, C10.0, C10.2-10.4, C10.8-10.9 (other oropharynx). Tumor histology was limited to ICD-0 codes for squamous histology $[\mathrm{n}=21,796 ; 805$ (papillary carcinoma, NOS), 807 (squamous cell carcinoma NOS), and 808 (lymphoepthelial carcinoma)]. Patients with data missing on sex $(n=0)$, hospital identification $(n=0)$, geographic region $(n=0)$, and HPV status 
$(n=13,437)$ were excluded. The analytic cohort comprised of a total of 8,359 patients who met the aforementioned study criteria.

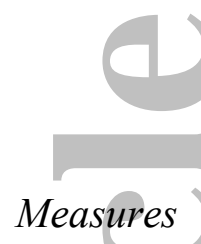

Patient-level independent variables included age at diagnosis, sex, race, ethnicity, comorbidity score, household income, educational attainment, and insurance status. Patient race was categorized by the NCDB as white, black, Asian/Pacific Islander, and Native American. Due to small numbers Asian/Pacific Islander and Native American were collapsed into an "other" category. Patient ethnicity was categorized independent of race as either non-Hispanic or Hispanic. The Charlson-Deyo comorbidity score was used to identify comorbid conditions within the cohort. Household income and educational attainment are area-level measures based on the patient's zip code at time of diagnosis with data drawn from the 2000 US census. Insurance status was defined by the patient's primary insurance carrier at the time of diagnosis and/or treatment and categorized as private including government, Medicare, Medicaid, and uninsured. Geographic location was determined by the location of the diagnosing facility within the 4 designated US census regions.

Primary tumor $(\mathrm{T})$, nodal $(\mathrm{N})$, and metastasis $(\mathrm{M})$ classification was categorized clinically according to the definitions used by the American Joint Committee on Cancer (AJCC), seventh edition. In cases where clinical $\mathrm{T}$ and/or N classification was either missing or unknown (11.5\% and $5.0 \%$ for $\mathrm{T}$ - and $\mathrm{N}$-, respectively), pathologic staging was used. Stage was recoded into stage 
I, II, III, IVA/B, and IVC using the American Joint Committee on Cancer's (AJCC) AJCC Cancer Staging Manual, $7^{\text {th }}$ edition.

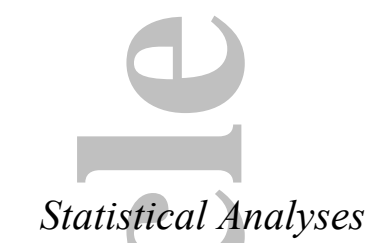

Distributions of clinical, non-clinical, and tumor characteristics between HPV-positive and HPVnegative tumors were compared using $\chi^{2}$ test for categorical variables and t test for continuous variables. Multivariable logistic regression was used to determine factors associated with HPV status. Because of substantial missingness in tumor grade, we conducted analyses both with and without tumor grade as a covariate in the model, and the results were similar. Multicollinearity was assessed using the generalized variance inflation factor (GVIF). All statistical tests were performed using R 3.1.1. The GVIFs were calculated in R with R package "Car". Two sided tests yielding $\mathrm{p}<0.05$ were considered as statistically significant.

\section{Results}

A total of 21,796 patients with OPC were identified before applying the exclusion criteria. The final analytic cohort consisted of 8,359 patients diagnosed with squamous cell carcinoma of the oropharynx between 2010 and 2011.

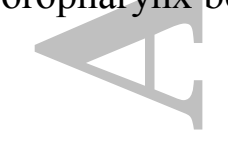

Table 1 summarizes the clinical, non-clinical, and tumor characteristics of the study population and distribution by tumor HPV status. The mean age was 58.4 years (range, 20 to 90 years). The overwhelming majority of patients were male $(82.2 \%)$, white $(91.4 \%)$, non-Hispanic $(96.8 \%)$, 
and had no associated comorbidity (83.0\%). Tumors typically arose within the base of tongue or tonsils (91.3\%) and most patients presented with locally advanced stage III/IV disease (85\%).

The frequency of HPV-related OPC in the present cohort was $65.4 \%$. The proportion of HPVrelated OPC was highest among young, white, non-Hispanic, males. Among patients ages 18 to 49 with OPC, HPV-related disease was observed in $69.7 \%$ compared to $68.5 \%$ among patients ages 50 to 64 and 55.2\% among patients ages 65 and older. The proportion of HPV-related OPC was significantly higher among whites (67.4\%) as compared to blacks (43.1\%), Asians (46.8\%), and American Indians/Eskimos $(42.9 \%)(P<0.001)$. HPV-related OPC was also higher among non-Hispanics compared to Hispanics $(65.6 \%$ vs. $55.5 \%, P<0.001)$ and among men compared to women $(67.5 \%$ vs. $55.7 \%, P<0.001)$.

Compared to patients with HPV-negative tumors, patients with HPV-positive tumors were significantly younger (mean age 57.5 vs. 60.1 years, $P<0.001)$ and more likely to be male $(P<$ 0.001), white $(P<0.001)$, hold private insurance $(P<0.001)$, graduate from high school $(P<$ $0.001)$, and have a higher household income $(P<0.001)$ (figure 1). Geographically, patients with HPV-negative OPC were most likely to be diagnosed in the South.

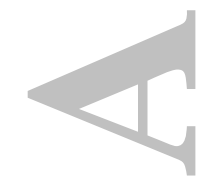

Compared to non-HPV-related OPC, HPV-positive tumors more commonly originated in the tonsil $(P<0.001)$ and were poorly differentiated $(P<0.001)$. Patients with HPV-positive tumors were more likely to present with earlier T-classification (T1-2, 76\% vs. 62\%; $P<0.001)$ and 
more advanced nodal disease $(\mathrm{N} 2-3,68 \%$ vs. $56 \% ; P<0.001)$ than patients with HPV-negative tumors (figure 2). Despite less advanced nodal disease, HPV-negative tumors were associated with a higher likelihood of metastatic disease at presentation $(4.2 \%$ vs. $1.7 \%, P<0.001)$.

In multivariable logistic regression analyses, age, sex, race, ethnicity, insurance status, education, geographic location, primary tumor site, tumor grade, and TNM classification remained significant determinants for HPV status (Table 2). Younger age and male sex were significantly associated with HPV-positive tumors (for younger age, 18 to 49 years odds ratio [OR], 1.39; $95 \%$ confidence interval [CI], 1.13-1.72; 50 to 64 years, OR, 1.34; 95\% CI 1.13-1.59; and for male sex, OR, 1.52; 95\% CI, 1.33-1.75). Non-white race (black race, OR, 0.54; 95\% CI, 0.440.66 and other race, OR, $0.49 ; 95 \%$ CI 0.33 to 0.73 ), Hispanic ethnicity (OR, $0.69 ; 95 \%$ CI, 0.51 0.93), absence of a high-school education ( $14 \%$ to $28.9 \%$ and $\geq 29 \%$ not graduating high school, OR $0.82 ; 95 \%$ CI, 0.72-0.94 and OR, 0.71; 95\% CI 0.58-0.88, respectively), and residence in the South (OR, 0.77; 95\% CI 0.67-0.89) were associated with HPV-negative tumors. Patients without private insurance were more likely to have HPV-negative tumors (Uninsured/Medicaid OR 0.50; 95\% CI, 0.43-0.59; Medicare OR, 0.74; 95\% CI, 0.63-0.88). There was a statistically significant difference in HPV status by primary site of origin within the oropharynx, with HPV negative tumors more likely to arise in the base of tongue (OR, 0.72; 95\% CI 0.65-0.81) and other oropharyngeal sites (OR, $0.37 ; 95 \%$ CI $0.31-0.45)$ as compared to the tonsils. Advanced primary tumor classification was associated with HPV-negative disease (T3, OR, 0.70; 95\% CI 0.59-0.82; T4, OR, 0.59; 95\% CI 0.50-0.71 [T1 = reference]), while nodal involvement was associated with HPV-positive disease (N1, OR 1.80, 95\% CI 1.52-2.14; N2, OR 2.25, 95\% CI 1.96-2.58; N3, OR 2.07, 95\% CI 1.55-2.77 [N0 = reference]). HPV-negative tumors were 
associated with a higher likelihood of metastatic disease at presentation (OR, 0.56, 95\% CI 0.39$0.79)$.

\section{Discussion}

The results of this study provide insight into the frequency and clinical presentation of HPVrelated orophargyneal cancer in the United States on a population level. Using a large, national hospital-based cancer registry, we observed an association with HPV in $65.4 \%$ of all newly diagnosed oropharyngeal carcinomas in 2010 and 2011 in the United States. HPV-positive tumors were associated with a distinct clinical profile, including younger age, male sex, white race, earlier primary tumor classification (T1-2), more advanced nodal classification (N2-3), and lower likelihood of metastatic disease at presentation.

While the increasing incidence of OPC has been well documented both in the United States ${ }^{1}$ and in other economically developed countries in North America ${ }^{2,3}$ and Western Europe, ${ }^{2,4-6}$ the true incidence of HPV-related OPC and associated clinical characteristics have not been well characterized on a national level. Retrospective analyses of clinical trial populations from 2002 to 2005 report HPV incidence rates of $57.3 \%$ and $63.8 \%$ among 185 and 323 patients with locally advanced, stage III to IV, OPC, respectively, ${ }^{9,10}$ whereas North American single institutional series have reported rates as high as $75 \%$ to $93 \% .^{20-22}$ Data from 3 Surveillance, Epidemiology, and End Results (SEER) registries in the United States demonstrate a substantial increase in HPV-related OPC from $16.3 \%$ during the 1980 s to $71.7 \%$ during the 2000 s. ${ }^{1}$ However, these rates were determined from a limited sample size, with HPV status available for 
only 271 tumor specimens over a 20 year period. ${ }^{1}$ Our study provides strong confirmation that the recent changes in the population-level incidence of OPC in the United States are attributable to HPV infection with an overall HPV frequency of $65.4 \%$ during 2010 to 2011 based on over 8,000 patients with newly diagnosed OPC. These findings provide the most representative data on HPV-related OPC for the US population to date.

In addition, our data extend prior retrospective observations from clinical trial populations 9,10 and single institutions ${ }^{7,13}$ that HPV-related OPC is a distinct clinical disease entity by showing that clinical differences persist on a population level between individuals with HPV-positive and negative disease. Consistent with other reports, we found younger age, male sex, and white race to be significantly associated with HPV-positive tumors. A major limitation of prior studies reporting racial differences in the percentage OPC attributable to HPV, primarily in blacks, is inadequate racial representation with the total number of black patients in these studies ranging from 28 to $49 .{ }^{1,14,23,24}$ Furthermore, these previous studies have provided information on the percentage of OPCs attributable to HPV in other racial groups such as Asians/Pacific Islanders or in patients of Hispanic ethnicity. Owing to our large sample size, we were able to show that the proportion of HPV-related OPC was significantly higher among whites (67.4\%) as compared to blacks (43.1\%), Asians (46.8\%), and American Indians/Eskimos (42.9\%). A similar finding was present between non-Hispanics (65.6\%) and Hispanics (55.5\%). These differences have been hypothesized to be due in part to racial variations in sexual behaviors with studies suggesting that a higher proportion of whites engange in oral sex, which has been linked to HPV transmission. ${ }^{13,25}$ However, it is important to note that the current epidemic of HPV-related OPC is not exclusive to white males. 
Geographically, we found that a disproportionate number of HPV-negative OPCs were located in the South, mirroring the higher rates tobacco and alcohol use in this region.

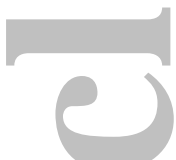

Patients with HPV-positive OPC were more likely to present with small primary tumors (T1-2: $75.6 \%$ vs. $62.2 \%$ ) and more advanced nodal disease (N2-3: $67.8 \%$ vs $56.1 \%$ ), which is consistent with previously reported single institution data from Princess Margaret Hospital (T1-2: 56\% vs 49\%; N2b-3: $69 \%$ vs $46 \%)^{20}$ as well as secondary analysis of the Trans Tasman Radiation Oncology Group (TROG 02.02) series of 185 patients (T1-2: 37\% vs 15\%; N2-3 86\% vs 65\%). ${ }^{9}$ In contrast, in a series of 111 patients with locally advanced OPC enrolled on TAX-324, ${ }^{11}$ patients with HPV-positive carcinoma were more likely to have smaller primary tumors (T1-2: $49 \%$ vs $20 \%$ ), but there was not difference in nodal status between the two groups. A similar finding was present in patients enrolled on RTOG $0129 .{ }^{10}$ The discrepancy in nodal presentation among these various prospective trials likely stems from their restrictive eligibility criteria and thereby limits the generalizability of these studies to the OPC population at large. Our findings on the association of HPV-related OPC with earlier primary tumor classification (T1-2) and more advanced nodal classification (N2-3) provide the most representative data on the presentation of HPV-related OPC to date.

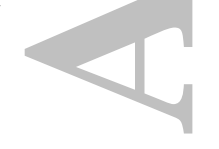

In addition, we found that despite more extensive nodal disease, patients with HPV-positive OPC were less likely to present with metastatic disease at diagnosis. It is unclear if this difference in the rate metastasis at presentation is attributable to tumor biology, delayed care secondary to 
socioeconomic disparities between patients with HPV-positive and negative tumors, or a combination of the two. For instance, we found that patients with HPV-negative tumors were more likely to be of black race, uninsured, and have a lower education level compared to those with HPV-positive tumors. Studies have found these factors to be associated with a greater likelihood of presenting with late-stage cancer at diagnosis across multiple disease sites. ${ }^{26,27}$ Following treatment, the rate of distant metastases between HPV-positive and HPV-negative tumors is similar, though these studies suggest that patients with HPV-positive OPC may develop metastases at a longer interval and have a higher proportion of the recurrences at distant sites. $^{28,29}$

C HPV status has recently been recognized as a significant independent prognostic factor in patients with OPC with multiple retrospective analyses of prospective clinical trials demonstrating improved overall survival and locoregional control for patients with locallyadvanced HPV-positive OPC compared to similarly treated patients with HPV-negative disease. $^{9-11,29}$ Overall survival for these patients with stage III-IV HPV-positive and HPVnegative tumors was $91 \%$ versus $74 \%$ (HR 0.36 ) at 2 -years on TROG $02.02^{9}$ and $82 \%$ versus $35 \%$ (HR 0.2) at 5-years on TAX $324 .{ }^{11}$ While there is currently insufficient information to alter therapy based on HPV status off clinical trial, treatment de-intensification is currently being explored for subpopulations of patients with locally-advanced HPV-positive OPC (T1-3, N0-2c) because of their favorable prognosis. ${ }^{20}$ Given the increasing incidence of OPC secondary to the HPV epidemic and the favorable prognosis of this younger, healthier population, developing strategies to optimize post-treatment quality of life (QOL) is imperative. 
There are several limitations to this study, most notably the number of exclusions due to missing HPV status. Ideally, the NCDB would have more complete representation of the variables included within the database. The adequacy of the NCDB for evaluating clinical characteristics in head and neck oncology on a national level might be evaluated in a separate dedicated analysis to fully explore the quality of the data and the influence of the large amount of missing data. Second, hospital differences in the method of HPV detection may yield discrepant results, depending on whether in-situ hybridization or immunohistochemistry was used. ${ }^{30}$ The NCDB does not provide information on the HPV detection method. However, it should be noted that national consensus on assay methodology is lacking. Finally, owing to the nature of data reporting in the NCDB, survival outcomes for the patients included in the present study are unavailable, limiting our ability to evaluate the impact of differences in HPV-status on clinical outcomes on a national level. These limitations are countered by the strengths of the study including its large size, national sample, and availability of information on HPV-status.

The results of this study show that HPV now accounts for the majority of newly diagnosed OPCs in the United States and that HPV-related OPC is associated with a distinct clinical profile, including younger age, male sex, white race, earlier primary tumor classification, more advanced nodal classification, and a lower likelihood of distant metastatic disease at presentation. However, it is important to note that the current epidemic of HPV-related OPC is not limited to white men. These findings have important implications for cancer prevention strategies, 
including prophylactic HPV vaccination, and lend support for efforts to re-evaluate the staging ${ }^{31}$ ${ }^{32}$ and treatment paradigm ${ }^{20,33}$ for HPV-associated oropharyngeal cancer.

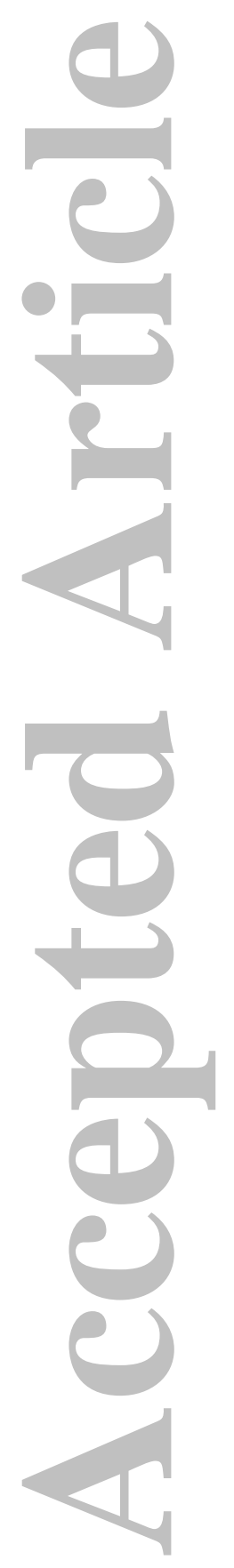

John Wiley \& Sons

This article is protected by copyright. All rights reserved. 


\section{Figure Legends}

Figure 1. Patient characteristics stratified by human papillomavirus (HPV) status.

Figure 2. Clinical TNM presentation stratified by human papillomavirus (HPV) status. (A)

Primary tumor classification. (B) Nodal classification. (C) Metastasis classification.
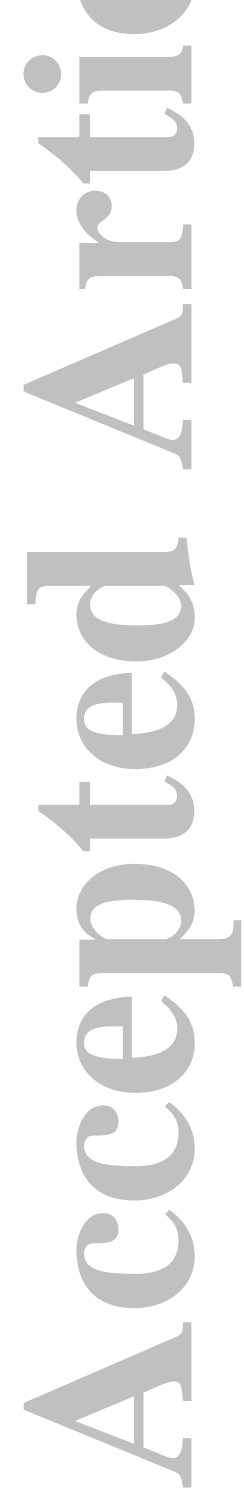

John Wiley \& Sons

This article is protected by copyright. All rights reserved. 


\section{References}

1. Chaturvedi AK, Engels EA, Pfeiffer RM, et al: Human papillomavirus and rising oropharyngeal cancer incidence in the United States. J Clin Oncol 29:4294-4301, 2011

2. Chaturvedi AK, Anderson WF, Lortet-Tieulent J, et al: Worldwide trends in incidence rates for oral cavity and oropharyngeal cancers. J Clin Oncol 31:4550-4559, 2013

3. Auluck A, Hislop G, Bajdik C, et al: Trends in oropharyngeal and oral cavity cancer incidence of Human Papillomavirus (HPV)-related and HPV-unrelated sites in a multicultural population: The British Columbia experience. Cancer 116:2635-2644, 2010

4. Hammarstedt $L$, Lindquist $D$, Dahlstrand $H$, et al: Human papillomavirus as a risk factor for the increase in incidence of tonsillar cancer. Int J Cancer 119:2620-2623, 2006

5. Braakhuis BJM, Visser O, René Leemans C: Oral and oropharyngeal cancer in The Netherlands between 1989 and 2006: Increasing incidence, but not in young adults. Oral Oncol 45:e85-e89, 2009

6. Conway DI, Stockton DL, Warnakulasuriya KAAS, et al: Incidence of oral and oropharyngeal cancer in United Kingdom (1990-1999)-recent trends and regional variation. Oral Oncol 42:586-592, 2006

7. Gillison ML: Evidence for a Causal Association Between Human Papillomavirus and a Subset of Head and Neck Cancers. J Natl Cancer Inst 92:709-720, 2000

8. Mehanna H, Beech T, Nicholson T, et al: Prevalence of human papillomavirus in oropharyngeal and nonoropharyngeal head and neck cancer-systematic review and meta-analysis of trends by time and region. Head Neck 35:747-755, 2013

9. Rischin D, Young RJ, Fisher R, et al: Prognostic significance of p16INK4Aand human papillomavirus in patients with oropharyngeal cancer treated on TROG 02.02 phase III trial. J Clin Oncol 28:4142-4148,

John Wiley \& Sons

This article is protected by copyright. All rights reserved. 
10. Kian Ang K, Ang KK, Harris J, et al: Human Papillomavirus and Survival of Patients with Oropharyngeal Cancer. N Engl J Med 363:24-35, 2010

11. Posner MR, Lorch JH, Goloubeva O, et al: Survival and human papillomavirus in oropharynx cancer in TAX 324: A subset analysis from an international phase III trial. Ann Oncol 22:1071-1077, 2011

12. Fakhry C, Westra WH, Li S, et al: Improved survival of patients with human papillomavirus-positive head and neck squamous cell carcinoma in a prospective clinical trial. J Natl Cancer Inst 100:261-269, 2008

13. Gillison ML, D'Souza G, Westra W, et al: Distinct risk factor profiles for human papillomavirus type 16-positive and human papillomavirus type 16-negative head and neck cancers. J Natl Cancer Inst $100: 407-420,2008$

14. Settle K, Posner MR, Schumaker LM, et al: Racial survival disparity in head and neck cancer results from low prevalence of human papillomavirus infection in black oropharyngeal cancer patients. Cancer Prev Res 2:776-781, 2009

15. Näsman A, Attner P, Hammarstedt L, et al: Incidence of human papillomavirus (HPV) positive tonsillar carcinoma in Stockholm, Sweden: An epidemic of viral-induced carcinoma? Int J Cancer 125:362-366, 2009

16. Lassen P, Eriksen JG, Krogdahl A, et al: The influence of HPV-associated p16-expression on accelerated fractionated radiotherapy in head and neck cancer: Evaluation of the randomised DAHANCA 6\&7 trial. Radiother Oncol 100:49-55, 2011

17. Ryerson AB, Peters ES, Coughlin SS, et al: Burden of potentially human papillomavirus-associated

John Wiley \& Sons

This article is protected by copyright. All rights reserved. 
cancers of the oropharynx and oral cavity in the US, 1998-2003. Cancer 113:2901-2909, 2008

18. Chaturvedi AK, Engels EA, Anderson WF, et al: Incidence trends for human papillomavirus-related and -unrelated oral squamous cell carcinomas in the United States. J Clin Oncol 26:612-619, 2008

19. Bilimoria KY, Stewart AK, Winchester DP, et al: The National Cancer Data Base: A Powerful Initiative to Improve Cancer Care in the United States. Ann Surg Oncol 15:683-690, 2008

20. O'Sullivan B, Huang SH, Siu LL, et al: Deintensification candidate subgroups in human papillomavirusrelated oropharyngeal cancer according to minimal risk of distant metastasis. J Clin Oncol 31:543-550, 2013

21. Lin BM, Wang H, D'Souza G, et al: Long-term prognosis and risk factors among patients with HPVassociated oropharyngeal squamous cell carcinoma. Cancer 119:3462-3471, 2013

22. Vainshtein JM, Spector ME, McHugh JB, et al: Refining risk stratification for locoregional failure after chemoradiotherapy in human papillomavirus-associated oropharyngeal cancer. Oral Oncol 50:513-519, 2014

23. Worsham MJ, Stephen JK, Chen KM, et al: Improved survival with HPV among african americans with oropharyngeal cancer. Clin Cancer Res 19:2486-2492, 2013

24. Zevallos JP, Sandulache VC, Hamblin J, et al: Impact of race on oropharyngeal squamous cell carcinoma presentation and outcomes among veterans. Head Neck 38:44-50, 2016

25. D’Souza G, Agrawal Y, Halpern J, et al: Oral Sexual Behaviors Associated with Prevalent Oral Human Papillomavirus Infection. J Infect Dis 199:1263-1269, 2009

26. Roetzheim RG, Pal N, Tennant C, et al: Effects of health insurance and race on early detection of cancer. J Natl Cancer Inst 91:1409-1415, 1999

John Wiley \& Sons

This article is protected by copyright. All rights reserved. 
27. Chen AY, Schrag NM, Halpern MT, et al: The impact of health insurance status on stage at diagnosis of oropharyngeal cancer. Cancer 110:395-402, 2007

28. Huang SH, Perez-Ordonez B, Weinreb I, et al: Natural course of distant metastases following radiotherapy or chemoradiotherapy in HPV-related oropharyngeal cancer. Oral Oncol 49:79-85, 2013

29. Nguyen-Tan PF, Zhang $Q$, Ang KK, et al: Randomized phase III trial to test accelerated versus standard fractionation in combination with concurrent cisplatin for head and neck carcinomas in the radiation therapy oncology group 0129 trial: Long-term report of efficacy and toxicity. J Clin Oncol 32:3858-3867, 2014

30. Jordan RC, Lingen MW, Perez-Ordonez B, et al: Validation of Methods for Oropharyngeal Cancer HPV Status Determination in US Cooperative Group Trials. Am J Surg Pathol 36:945-954, 2012

31. Sturgis EM, Dahlstrom KR, Garden AS, et al: Proposed Staging System for Patients With HPV-Related Oropharyngeal Cancer Based on Nasopharyngeal Cancer N Categories. J Clin Oncol 34:1848-54, 2016

32. Huang SH, Xu W, Waldron J, et al: Refining American joint committee on cancer/union for international cancer control TNM stage and prognostic groups for human papillomavirus-related oropharyngeal carcinomas. J Clin Oncol 33:836-845, 2015

33. Quon H, Forastiere AA: Controversies in treatment deintensification of human papillomavirusassociated oropharyngeal carcinomas: should we, how should we, and for whom? J Clin Oncol 31:520-2, 2013

John Wiley \& Sons

This article is protected by copyright. All rights reserved. 
Table 1. Distribution of patient and tumor characteristics by HPV status

\begin{tabular}{|c|c|c|c|c|c|c|c|}
\hline & \multicolumn{2}{|c|}{$\begin{array}{c}\text { Overall } \\
(\mathrm{n}=8359)\end{array}$} & \multicolumn{2}{|c|}{$\begin{array}{c}\text { HPV-positive } \\
(\mathrm{n}=5466,65.4 \%)\end{array}$} & \multicolumn{2}{|c|}{$\begin{array}{c}\text { HPV-negative } \\
(\mathrm{n}=2893,34.6 \%)\end{array}$} & \multirow[b]{2}{*}{$\mathrm{P}$ value* } \\
\hline & $N$ & $\%$ & $N$ & $\%$ & $N$ & $\%$ & \\
\hline \multicolumn{8}{|l|}{ Patient characteristics } \\
\hline Sex & & & & & & & $<0.001$ \\
\hline Male & 6869 & 82.2 & 4636 & 84.8 & 2233 & 77.2 & \\
\hline Female & 1490 & 17.8 & 830 & 15.2 & 660 & 22.8 & \\
\hline Age, $y$ & & & & & & & $<0.001$ \\
\hline $18-49$ & 1474 & 17.6 & 1028 & 18.8 & 446 & 15.4 & \\
\hline $50-64$ & 4781 & 57.2 & 3276 & 59.9 & 1505 & 52.0 & \\
\hline$\geq 65$ & 2104 & 25.2 & 1162 & 21.3 & 942 & 32.6 & \\
\hline Race & & & & & & & $<0.001$ \\
\hline White & 7553 & 91.4 & 5083 & 94.2 & 2470 & 86.2 & \\
\hline Black & 575 & 7.0 & 248 & 4.6 & 327 & 11.4 & \\
\hline Other & 132 & 1.6 & 63 & 1.2 & 69 & 2.4 & \\
\hline Ethnicity & & & & & & & $<0.001$ \\
\hline Non-hispanic & 7761 & 96.8 & 5093 & 97.3 & 2668 & 95.9 & \\
\hline Hispanic & 254 & 3.2 & 141 & 2.7 & 113 & 4.1 & \\
\hline Charlson-deyo comorbidity score & & & & & & & $<0.001$ \\
\hline 0 & 6935 & 83.0 & 4613 & 84.4 & 2322 & 80.3 & \\
\hline 1 & 1133 & 13.6 & 690 & 12.6 & 443 & 15.3 & \\
\hline$\geq 2$ & 291 & 3.5 & 163 & 3.0 & 128 & 4.4 & \\
\hline Insurance & & & & & & & $<0.001$ \\
\hline Uninsured & 396 & 4.8 & 203 & 3.8 & 193 & 6.8 & \\
\hline Medicaid & 639 & 7.8 & 315 & 5.9 & 324 & 11.4 & \\
\hline Medicare & 2159 & 26.2 & 1207 & 22.4 & 952 & 33.5 & \\
\hline Private & 5037 & 61.2 & 3662 & 68.0 & 1375 & 48.4 & \\
\hline \multicolumn{8}{|l|}{ Education } \\
\hline$<14 \%$ not graduating high school & 3215 & 41.2 & 2257 & 44.3 & 958 & 35.3 & $<0.001$ \\
\hline $14 \%-28.9 \%$ not graduating high school & 3562 & 45.6 & 2284 & 44.9 & 1278 & 47.0 & \\
\hline$\geq 29 \%$ not graduating high school & 1031 & 13.2 & 549 & 10.8 & 482 & 17.7 & \\
\hline Household income & & & & & & & $<0.001$ \\
\hline$<\$ 30,000$ & 888 & 11.4 & 474 & 9.3 & 414 & 15.2 & \\
\hline$\$ 30,000-45,95$ & 3438 & 44.0 & 2206 & 43.3 & 1232 & 45.3 & \\
\hline$\$ 46,000+$ & 3482 & 44.6 & 2410 & 47.4 & 1072 & 39.4 & \\
\hline Geographic location & & & & & & & $<0.001$ \\
\hline Northeast & 1915 & 22.9 & 1272 & 23.3 & 643 & 22.2 & \\
\hline Midwest & 2125 & 25.4 & 1496 & 27.4 & 629 & 21.7 & \\
\hline South & 2990 & 35.8 & 1788 & 32.7 & 1202 & 41.6 & \\
\hline West & 1329 & 15.9 & 910 & 16.7 & 419 & 14.5 & \\
\hline \multicolumn{8}{|l|}{ Tumor characteris } \\
\hline Year of diagnosis & & & & & & & 0.043 \\
\hline 2010 & 3182 & 38.1 & 2124 & 38.9 & 1058 & 36.6 & \\
\hline 2011 & 5177 & 61.9 & 3342 & 61.1 & 1835 & 63.4 & \\
\hline Primary site & & & & & & & $<0.001$ \\
\hline Base of tongue & 3407 & 40.8 & 2167 & 39.7 & 1240 & 42.9 & \\
\hline Tonsil & 4224 & 50.5 & 2987 & 54.6 & 1237 & 42.8 & \\
\hline Other oropharyngeal site & 728 & 8.7 & 312 & 5.7 & 416 & 14.4 & \\
\hline Grade & & & & & & & $<0.001$ \\
\hline Well differentiated & 255 & 4.0 & 113 & 2.7 & 142 & 6.4 & \\
\hline Moderately differentiated & 2722 & 42.3 & 1629 & 38.8 & 1093 & 48.9 & \\
\hline Poorly differentiated & 3455 & 53.7 & 2454 & 58.5 & 1001 & 44.8 & \\
\hline$T$ classification & & & & & & & $<0.001$ \\
\hline $\mathrm{T} 1$ & 2539 & 31.8 & 1818 & 34.6 & 721 & 26.4 & \\
\hline $\mathrm{T} 2$ & 3132 & $\begin{array}{c}39.3 \\
\text { John W }\end{array}$ & $\begin{array}{l}2154 \\
\& \text { Sons }\end{array}$ & 41.0 & 978 & 35.8 & \\
\hline
\end{tabular}


T3

T4

$\mathrm{N}$ classification

N0

N1

$\mathrm{N} 2$

N3

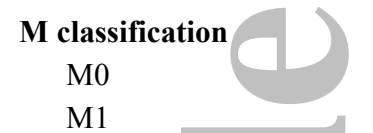

AJCC stage

I

II

III

IVA/B

IVC
1305

1004

1447

1528

4900

330

8088

213

441

550

1568

5222

213
16.4

12.6

17.6

18.6

59.7

4.0

97.4

2.6

5.5

6.9

19.6

65.3

2.7
772

505

719

1015

3443

203

5345

92

217

301

1035

3607

92
14.7

9.6

13.4

18.9

64.0

3.8

98.3

1.7

4.1

5.7

19.7

68.7

1.7
533

499

728

513

1457

127

2743

121

224

249

533

1615

121
19.5

18.3

25.8

18.2

51.6

4.5

$<0.001$

95.8

4.2

$<0.001$

Variables with missing data are as follows: race $(n=99 ; 1.2 \%)$, ethnicity $(n=344 ; 4.1 \%)$, insurance $(n=128 ; 1.5 \%)$, household income $(n=551$; $6.6 \%)$, education $(n=551 ; 6.6 \%)$, tumor grade $(n=1927 ; 23 \%)$, primary tumor classification $(n=379,4.5 \%)$, nodal classification $(n=154,1.8 \%)$, metastasis classification $(\mathrm{n}=58,0.7 \%)$, stage $(\mathrm{n}=365,4.4 \%)$.

Missing data were similarly distributed between HPV+ and HPV- patients for all variables.

*Missing data were excluded from P-value calculations.

Abbreviations: HPV, human papillomavirus; AJCC, American Joint Committee on Cancer

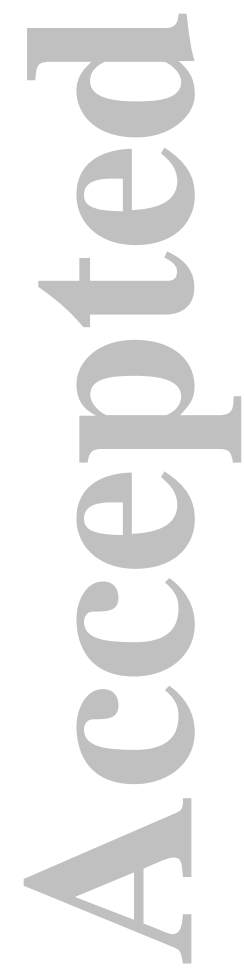


Table 2. Multivariable analysis of factors associated with HPV status

\begin{tabular}{|c|c|c|c|c|c|c|}
\hline \multirow[b]{2}{*}{ Variable } & \multicolumn{3}{|c|}{ Unadjusted } & \multicolumn{3}{|c|}{ Adjusted } \\
\hline & OR & $95 \% \mathrm{CI}$ & $P$ value & OR & $(95 \% \mathrm{CI})$ & $P$ value \\
\hline \multicolumn{7}{|l|}{ Sex } \\
\hline Male & 1 & [Reference] & & 1 & [Reference] & \\
\hline Female & 0.57 & $(0.50-0.65)$ & $<0.001$ & 0.66 & $(0.57-0.75)$ & $<0.001$ \\
\hline \multicolumn{7}{|l|}{ Age, $y$} \\
\hline $18-49$ & 1.79 & $(1.53-2.09)$ & $<0.001$ & 1.39 & $(1.13-1.72)$ & 0.002 \\
\hline $50-64$ & 1.74 & $(1.55-1.96)$ & $<0.001$ & 1.34 & $(1.13-1.59)$ & 0.001 \\
\hline$\geq 65$ & 1 & [Reference] & & 1 & [Reference] & \\
\hline \multicolumn{7}{|l|}{ Race } \\
\hline White & 1 & [Reference] & & 1 & [Reference] & \\
\hline Black & 0.37 & $0.30-0.44$ & $<0.001$ & 0.54 & $(0.44-0.66)$ & $<0.001$ \\
\hline Other & 0.46 & $0.31-0.67$ & $<0.001$ & 0.49 & $(0.33-0.73)$ & 0.001 \\
\hline \multicolumn{7}{|l|}{ Ethnicity } \\
\hline Non-hispanic & 1 & [Reference] & & 1 & [Reference] & \\
\hline Hispanic & 0.62 & $0.47-0.82$ & 0.001 & 0.69 & $(0.51-0.93)$ & 0.013 \\
\hline \multicolumn{7}{|l|}{ Charlson-deyo comorbidity score } \\
\hline 0 & 1 & [Reference] & & 1 & [Reference] & \\
\hline$\geq 1$ & 0.73 & $(0.64-0.83)$ & $<0.001$ & 0.94 & $(0.91-1.08)$ & 0.37 \\
\hline \multicolumn{7}{|l|}{ Insurance } \\
\hline Private & 1 & [Reference] & & 1 & [Reference] & \\
\hline Medicare & 0.48 & $(0.43-0.54)$ & $<0.001$ & 0.74 & $(0.63-0.88)$ & $<0.001$ \\
\hline Uninsured/Mec & 0.36 & $(0.31-0.42)$ & $<0.001$ & 0.50 & $(0.43-0.59)$ & $<0.001$ \\
\hline \multicolumn{7}{|l|}{ Education } \\
\hline$<14 \%$ not graduating high school & 1 & [Reference] & & 1 & [Reference] & \\
\hline $14 \%-28.9 \%$ not graduating high school & 0.75 & $(0.68-0.84)$ & $<0.001$ & 0.82 & $(0.72-0.94)$ & 0.005 \\
\hline$\geq 29 \%$ not graduating high school & 0.49 & $(0.42-0.58)$ & $<0.001$ & 0.71 & $(0.58-0.88)$ & 0.002 \\
\hline \multicolumn{7}{|l|}{ Household income } \\
\hline$<\$ 30,000$ & 0.52 & $(0.44-0.61)$ & $<0.001$ & 1.00 & $(0.80-1.25)$ & 0.99 \\
\hline$\$ 30,000-45,999$ & 0.82 & $(0.73-0.91)$ & $<0.001$ & 1.09 & $(0.95-1.26)$ & 0.23 \\
\hline$\$ 46,000+$ & 1 & [Reference] & & 1 & [Reference] & \\
\hline \multicolumn{7}{|l|}{ Facility location } \\
\hline Northeast & 1 & [Reference] & & 1 & [Reference] & \\
\hline Midwest & 1.20 & $(1.04-1.39)$ & 0.014 & 1.10 & $(0.94-1.29)$ & 0.23 \\
\hline South & 0.75 & $(0.66-0.86)$ & $<0.001$ & 0.77 & $(0.67-0.89)$ & $<0.001$ \\
\hline West & 1.12 & $(0.95-1.32)$ & 0.19 & 1.03 & $(0.86-1.23)$ & 0.73 \\
\hline \multicolumn{7}{|l|}{ Primary site } \\
\hline Tonsil & 1 & [Reference] & & 1 & [Reference] & \\
\hline Base of tongue & 0.71 & $(0.64-0.79)$ & $<0.001$ & 0.72 & $(0.65-0.81)$ & $<0.001$ \\
\hline Other oropharyngeal site & 0.29 & $(0.24-0.35)$ & $<0.001$ & 0.37 & $(0.31-0.45)$ & $<0.001$ \\
\hline \multicolumn{7}{|l|}{ Grade } \\
\hline Well/moderately differentiated & 1 & [Reference] & & 1 & [Reference] & \\
\hline Poorly differentiated & 1.78 & $(1.59-2.00)$ & $<0.001$ & 1.62 & $(1.44-1.88)$ & $<0.001$ \\
\hline Unspecified & 1.46 & $(1.28-1.67)$ & $<0.001$ & 1.42 & $(1.23-1.64)$ & $<0.001$ \\
\hline \multicolumn{7}{|l|}{$T$ classification } \\
\hline $\mathrm{T} 1$ & 1 & [Reference] & & 1 & [Reference] & \\
\hline $\mathrm{T} 2$ & 0.88 & $(0.78-1.00)$ & 0.05 & 0.94 & $(0.82-1.07)$ & 0.34 \\
\hline $\mathrm{T} 3$ & 0.56 & $(0.48-0.66)$ & $<0.001$ & 0.70 & $(0.59-0.82)$ & $<0.001$ \\
\hline $\mathrm{T} 4$ & 0.40 & $(0.34-0.47)$ & $<0.001$ & 0.59 & $(0.50-0.71)$ & $<0.001$ \\
\hline \multicolumn{7}{|l|}{ N classification } \\
\hline N0 & 1 & [Reference] & & 1 & [Reference] & \\
\hline
\end{tabular}




\begin{tabular}{crrrrrr}
\hline N1 & 2.02 & $(1.71-2.38)$ & $<0.001$ & 1.80 & $(1.52-2.14)$ & $<0.001$ \\
N2 & 2.42 & $(2.12-2.76)$ & $<0.001$ & 2.25 & $(1.96-2.58)$ & $<0.001$ \\
N3 & 1.67 & $(1.28-2.19)$ & $<0.001$ & 2.07 & $(1.55-2.77)$ & $<0.001$ \\
M classification & & & & & & \\
M0 & 1 & {$[$ Reference $]$} & & 1 & {$[$ Reference $]$} & \\
M1 & 0.40 & $(0.29-0.56)$ & $<0.001$ & 0.56 & $(0.39-0.79)$ & 0.001 \\
\hline
\end{tabular}

Year of diagnosis did not demonstrate statistical significance on multivariable analysis (data not shown).

Abbreviations: HPV, human papillomavirus; OR, Odds Ratio; CI, Confidence interval
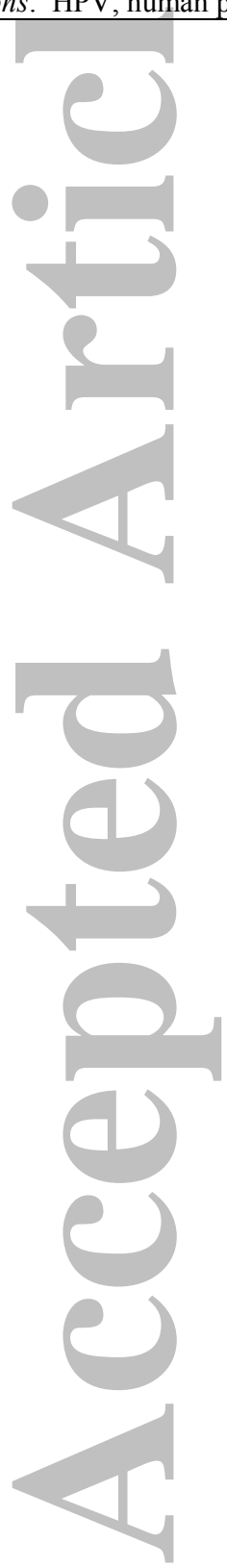


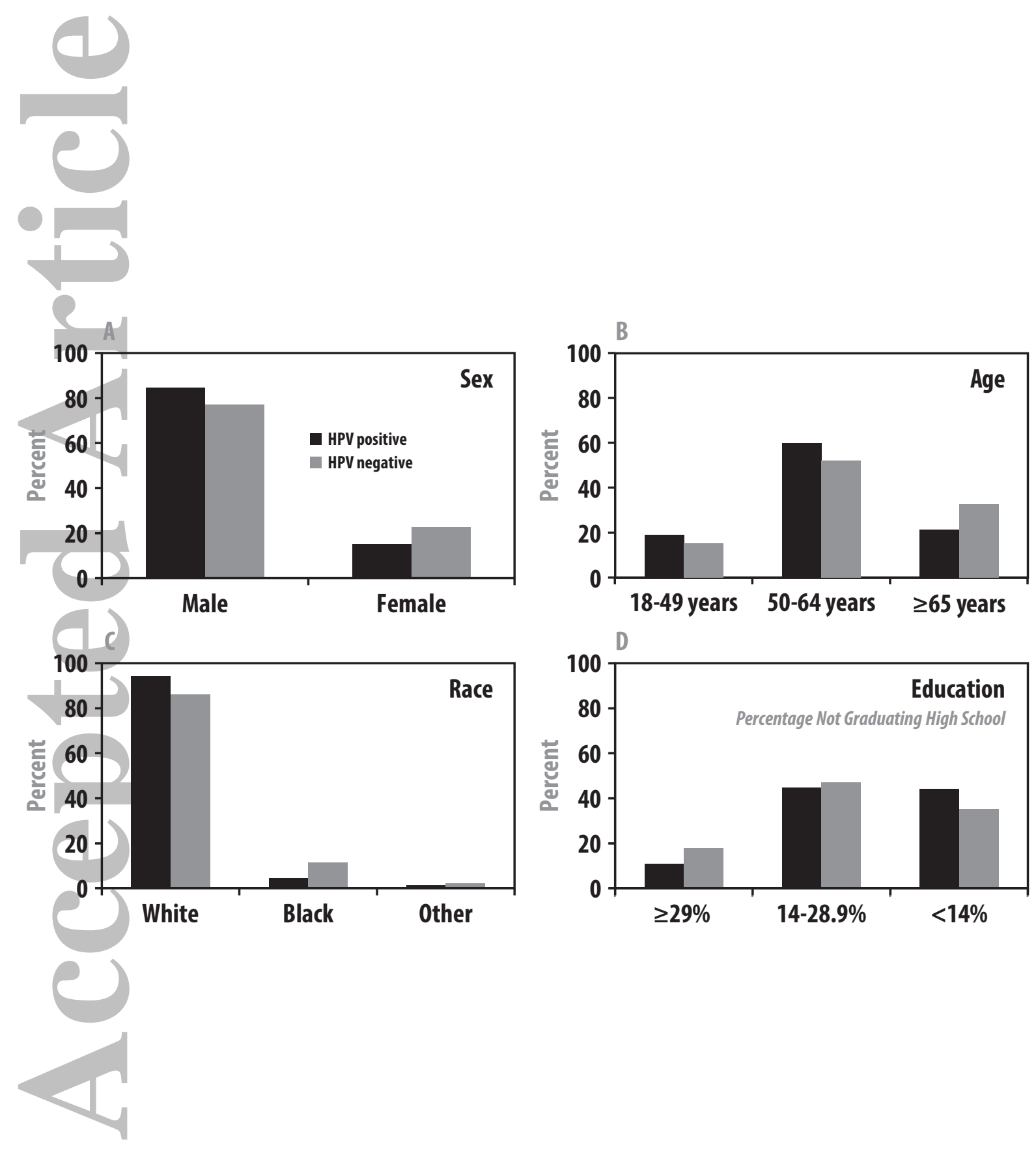

John Wiley \& Sons

This article is protected by copyright. All rights reserved. 


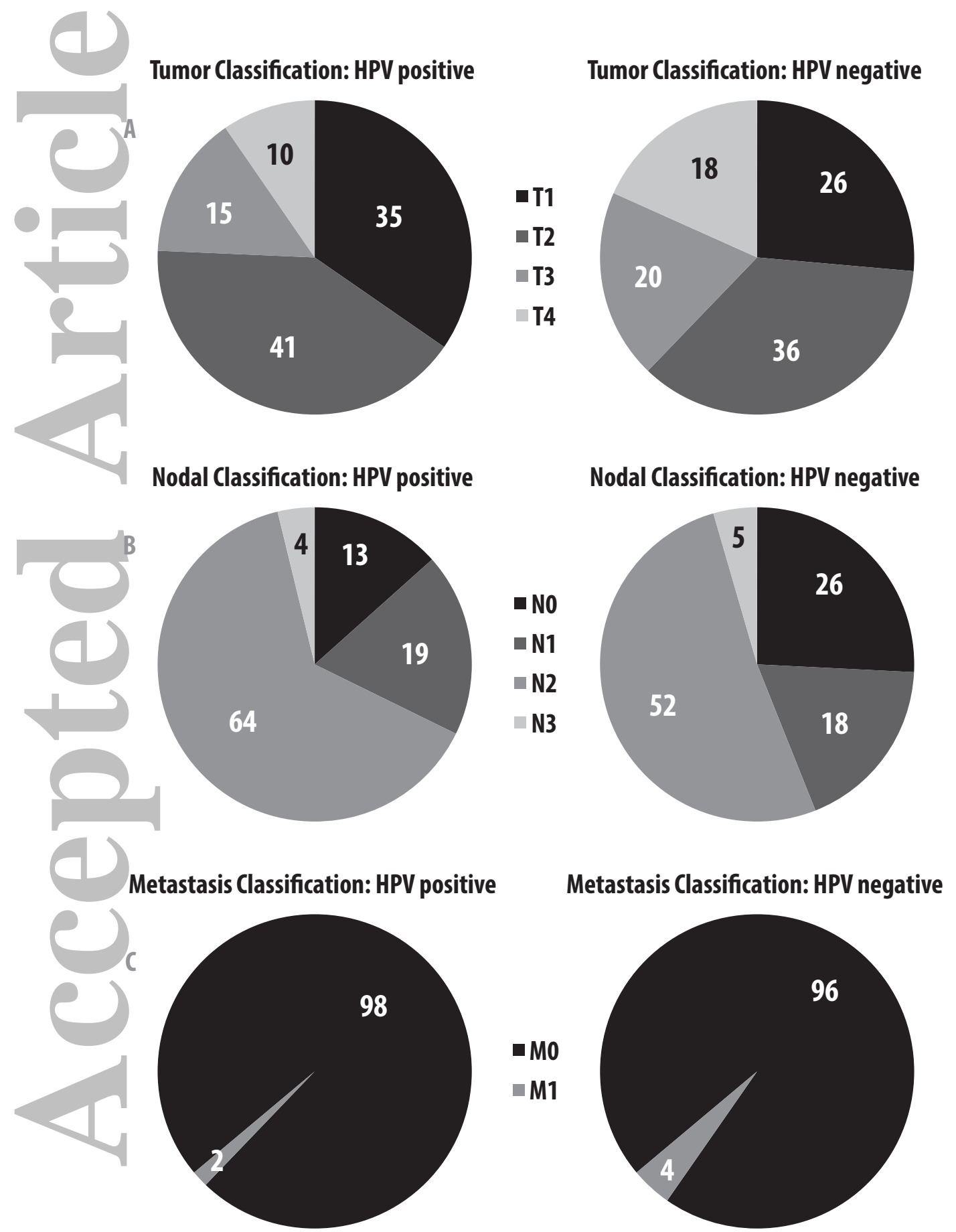

